\title{
Experiments on a dry granular avalanche impacting an obstacle: dead zone, granular jump and induced forces
}

\author{
P. Caccamo, T. Faug, H. Bellot \& F. Naaim-Bouvet \\ Cemagref, ETGR, St-Martin d'Hères, France
}

\begin{abstract}
This work describes small-scale laboratory tests on dry granular avalanches. Avalanches flow down a channel and impact a wall-like obstacle. A deposit generates upstream of the obstacle and plays an important role in the definition of the total mean force induced on the obstacle by the flow. The estimation of this force is crucial to design efficient protection structures against snow avalanches. Keywords: granular, avalanche, obstacle, force, stagnant zone, laboratory tests.
\end{abstract}

\section{Introduction}

Mountainous areas have often to take into account the snow avalanche hazard. Protection systems are designed even to prevent the flow to start (active protection) or to protect goods once the avalanche released (passive protection). Passive protection can be done by stopping the flow (catching dams), by deviating its course (deflecting dams) or by spreading the flow and dissipating its energy (breaking mounds). The optimal solution, for passive defence, depends on the location in the run-out zone and the shape of protection structures and on the force they have been designed to support. When a snow avalanche impacts an obstacle, a deposit generates upstream of the obstacle and plays an important role in the definition of the total mean force induced on the obstacle by the flow. This study aims at investigating the behavior of this deposit and its evolution from the beginning to the end of the flow. In Section 2 the experimental approach is introduced. First, an overview on the experimental device and on previous studies carried out on the same device is given, then the new adopted measurement techniques are described. Section 3 presents obtained results in terms of the 
influence zone upstream of the obstacle. Experimental data are presented and then used to extrapolate a theoretical prediction. Finally, Section 4 concludes this work showing its contribution to the knowledge on avalanche protection matter.

\section{Experimental approach}

This study is based on a small-scale experimental approach. This kind of approach allows the repetition of as many tests as wished in totally safe condition, and the control of characteristic parameters. Tests are based on the concept of measurement repeatability. Anyway, in order to avoid random errors due to experimental conditions, each test has been repeated five to ten times at constant parameters and the average value is presented as representative of the sample. A granular material has been chosen to reproduce dense snow avalanches. Analogies between snow and granular material have been previously underlined in [1,10-12].

\subsection{The device}

The experimental device is shown in Fig. $1 a$ and it consists in an inclined plane on which a channel has been mounted. The channel bottom is covered with sandpaper to introduce roughness. The inclination of the plane can be varied as well as the channel width and its length. For presented tests, the channel width and length are fixed and equal, respectively, to $0.25 \mathrm{~m}$ and $1.3 \mathrm{~m}$. On top of the channel a reservoir is mounted. It stores a constant mass $m=9.2 \mathrm{~kg}$ of granular material which can be released by opening a gate. The granular material consists in monodispersed glass beads of density $\rho_{p}=2450 \mathrm{~kg} / \mathrm{m}^{3}$ and mean diameter $d=1 \mathrm{~mm}$. Glass beads have been characterized with the function $h_{s t o p}(\vartheta)$ which defines the relevant friction angles of the material [7,9]. Tests have been performed by releasing a finite volume of material down the incline and observing the final deposit height $\left(h_{\text {stop }}\right)$ remaining on the bottom with respect to the channel slope. As shown in Fig.1c, the friction angles $\vartheta_{\text {min }}$ (inclination value for which $h_{\text {stop }}$ tends to infinite) and $\vartheta_{\max }$ (value for which $h_{\text {stop }}$ tends to zero) have been experimentally found to be close to $20^{\circ}$ and $28^{\circ} \cdot \vartheta_{\min }$ and $\vartheta_{\max }$ refer to the effective friction coefficients associated with quasi-static deformation and rapid collisional flows, respectively. As shown in Fig. 1c, note that these friction angles are larger in the 3D case compared to the 2D case (see 2D numerical study reported in $[4,5]$ ).

Performed tests aim to investigate the flow depth $h$, the flow velocity $U$ and the force $F$ that the flow can exert on an obstacle placed at a distance $x=1300 d$ from the releasing gate, in a range of slopes from $21^{\circ}$ (close to $\vartheta_{\text {min }}$ ) to $33^{\circ}$ (close to $\left.\vartheta_{\max }\right)$. Additionally, tools integrate the experimental device to carry out this kind of measurements. A high frequency video camera can be fixed normal to the flow surface or to the channel side (side walls are made of plexiglass). Each test is filmed and image sequences are then treated. A laser is installed on the device for flow depth measurements. For force measurements an obstacle can be mounted at the end of the channel in order to span the whole channel width and to measure the mean force exerted by the flow. Fig. $1 b$ shows the obstacle. It consists in a flat steel 
(a)

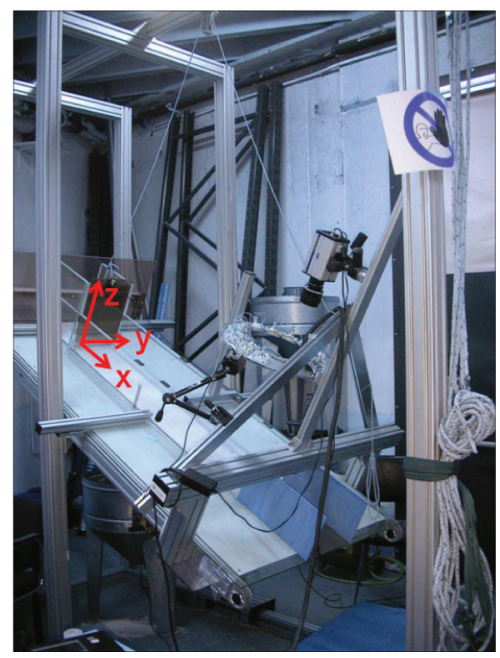

(b)

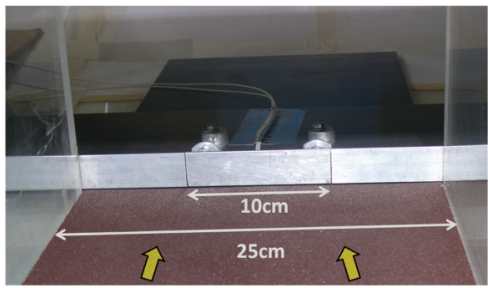

(c)

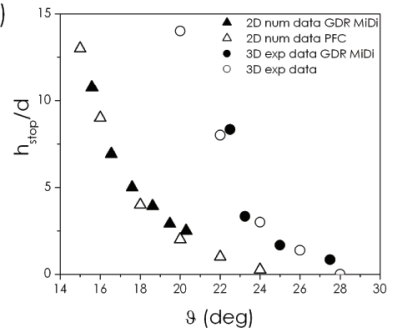

Figure 1: (a) Experimental device overview; (b) Front view of the obstacle. Yellow arrows indicate the direction of the incoming flow; (c) $h_{\text {stop }}$ behavior versus the slope angle $\vartheta$ for $2 \mathrm{D}$ and $3 \mathrm{D}$ case $[4,5]$.

beam of $2.5 \mathrm{~cm}$ height normal to the bottom and to the flow direction. Because of vibration and resonance problems, only the central $10 \mathrm{~cm}$ are connected to force sensors, the rest of the obstacle obstructs the channel without contributing to the force computation.

\subsection{Previous measurements and results}

The presented work focuses on some aspects of a wider frame. The previous study, realised on the same experimental device, aimed to characterise the flow from a dynamic point of view and to measure forces that the flow can exert. Reference tests were performed first. It consisted in releasing avalanches without any obstacle along the flow path and to measure the flow depth and the surface velocity evolution versus time at different slope angles $\left(\vartheta=21^{\circ}-33^{\circ}\right.$ each degree). Results are shown in Fig. $2 a$ and $b$. Then, thanks to the force sensors, the force exerted on the obstacle could be quantified for each inclination angle (see Fig.2c). The maximum force value has been retained and compared to two typical force contributions, the hydrostatic force $F_{h y d}$ and the dynamic one $F_{d y n}$ :

$$
\begin{gathered}
F_{h y d}=\frac{1}{2} \phi(t) \rho_{p} g h^{2}(t) \cos \vartheta \\
F_{d y n}=\frac{1}{2} \phi(t) \rho_{p} U^{2}(t) h(t)
\end{gathered}
$$


(a)

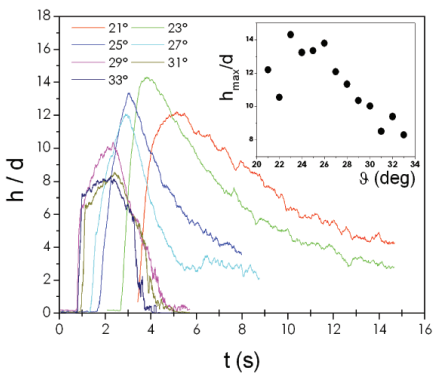

(c)

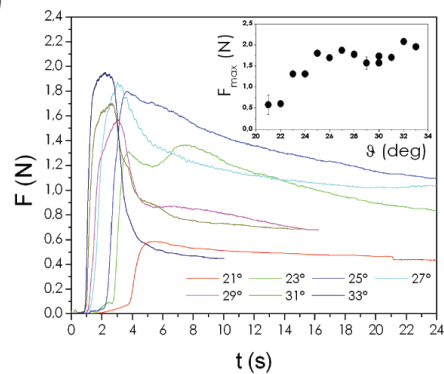

(b)

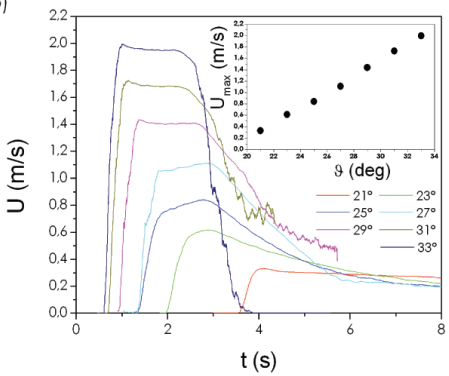

(d)

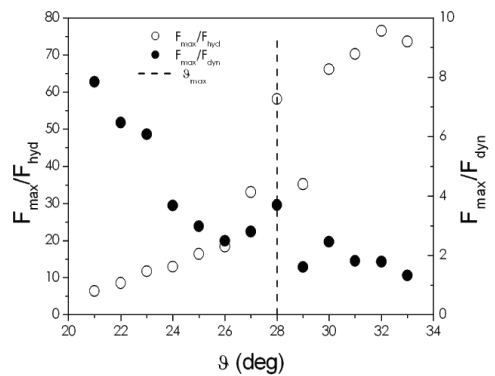

Figure 2: Reference measurements results. (a) Evolution of the flow height $h$ normalised by the beads diameter $d$ versus the time $t$. The insert shows the maximum values of $h / d$ versus the slope angle $\vartheta$; (b) surface velocity $U$ versus $t$. The insert shows the maximum $U$ values versus $\vartheta$; and (c) mean force $F$ versus $t$. The insert shows maximum $F$ values versus $\vartheta$. Measurements have been carried out at a distance $x=1300 d$ from the releasing gate. (d) Evolution of $F_{\max } / F_{d y n}$ and $F_{\max } / F_{\text {hyd }}$ ratios versus $\vartheta$.

where $t$ has been considered as $t_{h=h_{\max }}$ and the volume fraction $\phi$ derives from the scaling law relating $\phi$ to $U$ and $h$ [6]. Fig. $2 d$ shows the ratios $F_{\text {max }} / F_{h y d}$ and $F_{\text {max }} / F_{d y n}$ versus the slope angle $\vartheta$. Both the ratios underline a change in the regime occurring around $\vartheta_{\text {max }}$. Results match with numerical simulations carried out for the $2 \mathrm{D}$ case $[4,5] . F_{\max } / F_{d y n}$ tends to one for high slopes meaning that the maximum force is purely dynamic. For low slopes $F_{\max }$ is about 5-6 time $F_{\text {hyd }}$. A third contribution occurs and plays an important role in the total force. This contribution is given by the presence of the stagnant zone upstream the obstacle (see Fig. $3 b$ ) resulting from the flow/obstacle interaction. This is why the actual study aims to better understand the behavior of the stagnant zone.

\subsection{New measurements}

As mentioned above, previous studies investigated the flow characteristics at different slope angles. Now attention is focused on one slope angle $\vartheta=31^{\circ}$ (larger than $\vartheta_{\text {max }}$ ) and two main kinds of measurements have been carried out in 
presence of the wall-like obstacle, which allows to have the access to the freesurface modification in the vicinity of the wall. First, the laser has been added to the device and the high frequency video camera fixed at the end of the channel, normal to the bottom and to the main flow direction. Flow depth measurements have been coupled with force measurements. Then the video camera has been positioned on the side of the channel, on the same axis of the obstacle, normal to the flow direction. The flow has been recorded and the image sequences treated thanks to a Matlab routine.

\subsubsection{Flow depth measurements}

Flow depth measurements have been realised using a laser technique. A laser beam is projected along the channel axis and deviated by the incoming flow transit. The deviation $\Delta$ of the line is proportional to the flow height $h$. The video camera recorded each test at an acquisition frequency of 350fps. An accurate calibration is needed to relate the measured $\Delta$ values to the incoming flow depth. This calibration has been realised by using three standard plates in order to cover a calibration range of $0-24 \mathrm{~mm}$ thickness. The error on the laser line detection increases when increasing the dilution rate. For rather dense flows it is estimated to be less than $0.5 d$ whereas for very dilute flows it is difficult to define it.

\subsubsection{Force measurements}

Force measurements have been done thanks to two XFTC300 sensors connected to the obstacle. Sensors measure the mean force induced by the flow, acting along the $\mathrm{X}$-axis (see Fig.1a), as a tension signal [V]. A calibration is necessary to relate the detected tensions to force values. Calibration consisted of applying well known forces on the obstacle in order to derive the relatives measured tensions. The obstacle has been positioned horizontally and six weights have been loaded one on top of the other and off-loaded one by one. This procedure was repeated at five
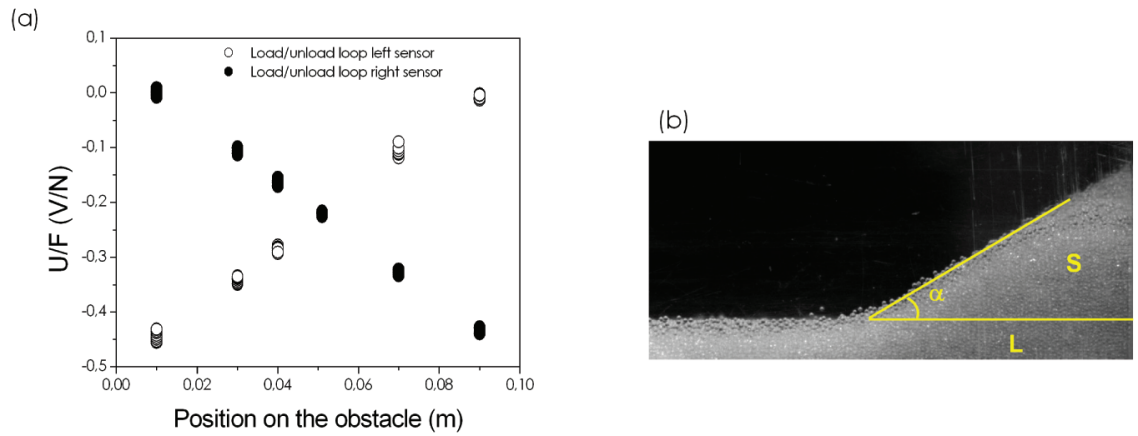

Figure 3: (a) Load/unload cycles representing the calibration procedure for force sensors. No hysteresis effects are evidenced; (b) Stagnant zone occurring upstream of the obstacle and the three parameters chosen to describe its temporal evolution. 
positions all along the upstream face of the obstacle. The load/unload cycle has been tested to find out potential hysteresis effects. Fig. $3 a$ shows that these effects are negligible. Two different kind of validations [2] has been carried out to test the reliability of the calibration. With those two methods, the maximum estimated error is equal to $21 \mathrm{mN}$ for a minimum calibration force of $0.65 \mathrm{~N}$, which corresponds to an error of $3 \%$.

\subsubsection{Image treatment}

Side view recordings allow the investigation of the parameters characterizing the evolution of the stagnant zone. With respect to Fig.3b, the stagnant zone is defined as the area upstream of the obstacle where the obstacle exerts its influence on the flow. To quantify its evolution, three parameters have been chosen: the length $L$ of the influence zone, the mean inclination angle $\alpha$ of the free surface and the involved surface $S$. The high speed video camera is set at an acquisition frequency of 350fps. Image treatment analyses each picture, detects the area occupied by the flow and derives the corresponding free surface profile. This profile allows the definition of the three parameters every $t=1 / 350 \mathrm{~s}$. The treatment has been validated by a cross comparison with data obtained for flow depth measurements realised with and without the obstacle thanks to the laser technique. The precision of this method depends, as for the flow depth case, on the dilution rate of the flow. The arrival and the final phase of the flow are characterised by a very dilute state where image treatment cannot be very reliable. For the rest of the flow the estimated error in the free surface detection is less than $2 d$, which corresponds to an error of less than $2 \%$ on the final deposit mean length.

\section{Results}

\subsection{Experimental data}

A first rough analysis of the obtained videos (side view) gives an impression that a transition occurs in the stagnant zone regime. Both realised measurements confirm it. Fig. $4 a$ shows the flow height $h$ along the x-axis upstream of the obstacle. Height values have been obtained with the laser technique. With respect to curves in Fig. $4 a$, at $0.003 \mathrm{~s}<t<1.3 \mathrm{~s}$ the avalanche front arrives, it impacts the obstacle and a jet occurs. Near $t=2 \mathrm{~s}$ a dead zone regime is attained. A quasi-static triangular zone forms upstream of the obstacle and the inertial flow starts flowing above. This inertial layer becomes thicker $(t=3 \mathrm{~s})$ and then thinner again before the transition starts. In fact, at $4 \mathrm{~s}<t<4$.7s the stagnant zone switches from a dead zone to a granular jump regime. Curves show a change in the stagnant zone shape. The triangular quasi-static zone develops into more of a trapezoidal surface (see Fig. $5 a$ ), increasing in volume and progressing upstream in the opposite direction of the flow until the motion stops. This new regime is characterized by a discontinuity in flow depth and surface velocity.

Fig. $4 b, c$ and $d$ show results obtained thanks to an accurate treatment of side view images. As already mentioned in section 2.3.3, three parameters have been 
a)

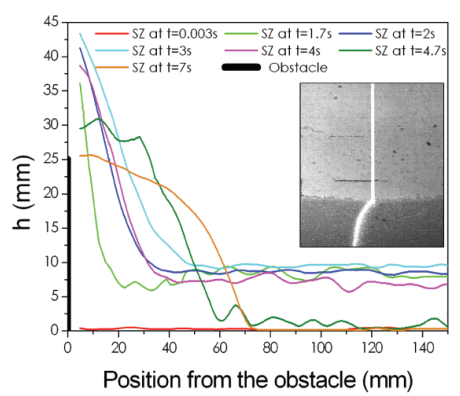

(c)

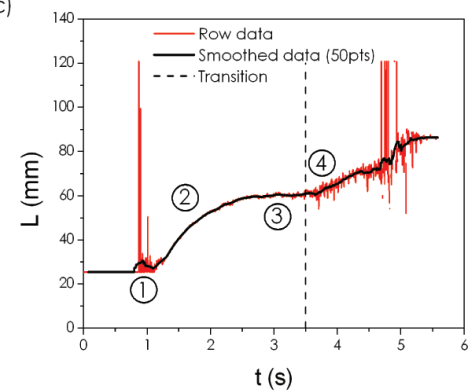

(b)

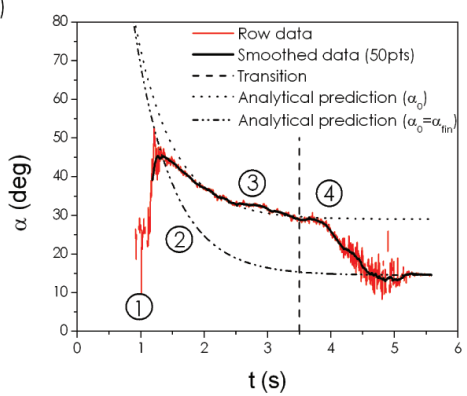

(d)

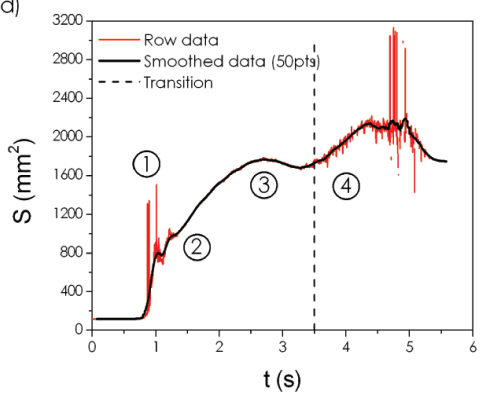

Figure 4: (a) Flow depth laser measurements along the x-axis upstream of the obstacle. The insert shows a typical image of the final deposit and of the laser line used for measurements. Results obtained from side-view measurements, at $\vartheta=31^{\circ}$, concerning: (b) the stagnant zone mean angle $\alpha$ versus the time $t$, (c) the length $L$ versus $t$ and (d) the surface $S$ versus $t$. The dashed line indicates the transition time derived from experimental data analysis.

chosen to describe the evolution of the stagnant zone (see Fig.3b): its mean slope $\alpha$, its length $L$ and its surface $S$. Figures show $\alpha, L$ and $S$ versus the time $t$. The flow phases that have been found with laser measurements can be here identified again with a better precision. Near $t=1$ s the front arrives on the obstacle (phase 1). The $\alpha$ angle attains very high values (jet effect) and $L$ and $S$ raw data fluctuate. At 1 s $<t<2$ s the stagnant zone generates (phase 2), the upstream deposit assumes a triangular shape, $\alpha$ decreases, $L$ and $S$ start increasing. The dead zone reaches a stationary regime around $2 \mathrm{~s}<t<3.5 \mathrm{~s}: \alpha$ and $L$ stabilize at constant value, $\mathrm{d} L / \mathrm{d} t=0$ (phase 3 ). $S$ has an increasing-decreasing phase due to the thickeningthinning process of the inertial layer flowing above the dead zone. After $t \simeq 3.7 \mathrm{~s}$ transition starts (phase 4). The hydraulic jump succeeds to the dead zone regime. The transition implies a decrease of $\alpha$ and an increase of $L$ until the flow stops. As the stagnant zone progresses upstream the channel, $S$ increases. A short decreasing of surface value follows just before the end of the motion due to the fact that the last 
portion of the avalanche volume flows down the channel eroding a part of the final volume. After $t \simeq 5.2 \mathrm{~s}$ the avalanche flow comes to a standstill. The final deposit, stored upstream of the obstacle, is then characterized by constant values of the three parameters corresponding to static condition. Additionally, Fig. $4 b$ shows the analytical predictions for the stagnant zone slope angle. An empirical exponential law defines the angle $\alpha$ as

$$
\alpha=\alpha_{0}+\left(\frac{\pi}{2}-\alpha_{0}\right) e^{\frac{T_{i}-t}{\tau}}
$$

(see for details [3]). $\alpha_{0}$ is the value of $\alpha$ attained for stationary regime $\left(\alpha_{0}=29^{\circ}\right.$ ), $T_{i}$ the time of impact $\left(T_{i}=0.8 \mathrm{~s}\right)$ and $\tau$ is a characteristics time of the flow ( $\tau=0.6 \mathrm{~s}$ ). This empirical law allows to reproduce the behavior of $\alpha$ during the formation of the stagnant zone until the stationary phase. If forcing the prediction to stabilize $\alpha$ at a value corresponding to the $\alpha$ angle of the final deposit $\left(\alpha_{\text {fin }}=14.5^{\circ}\right)$, the law is not reliable any more except for the final slope value. Fig. $4 b$ highlights that the difference between the final deposit value of $\alpha\left(\alpha_{f i n}\right)$ and the stationary value $\left(\alpha_{0}\right)$ is due to the observed transition from the dead zone to the granular jump regime.

\subsection{Theoretical analysis and prediction}

The dead zone to granular jump transition has been analysed more in details. According to the classical analysis of bi-dimensional hydraulic jump, the mass and momentum fluxes are conserved across the jump while the mechanical energy is dissipated [8]. Eq.(4) is obtained writing the mass and momentum conservation across a stationary jump in order to calculate the critical obstacle height which can generate a jump:

$$
\frac{H_{c}}{h}=\frac{1}{2}\left(\sqrt{1+8 F r^{2}}-1\right)-F r^{2 / 3}
$$

Three main assumptions are made: the density $\rho$ is constant, the incoming regime is stationary and friction dissipations are not considered (see details in [8]). This equation has been compared to the ratio $H_{o b s} / h$, where $H_{o b s}$ is the obstacle height $\left(H_{o b s}=0.025 \mathrm{~m}\right)$ and $h$ corresponds to experimental data obtained for reference flow depth at $\vartheta=31^{\circ}$. Fig. $5 b$ shows the ratios $H_{o b s} / h$ and $H_{c} / h$ versus the time $t$. The two curves cross at $t=3.3 \mathrm{~s}$, which roughly corresponds to the time $t *$ at which the dead zone to granular jump transition occurred in the side view video analysis. Fig. $5 c$ shows the mean force $F$ exerted on the obstacle versus the time $t$ at $\vartheta=31^{\circ}$. The curve is characterised by an increase in values till a maximum, preceding a decrease phase. A change in the decreasing trend can be observed near $t=t *$. This is probably due to the influence of the transition on the total force induced on the obstacle by the flow.

Using experimental data concerning reference tests carried out for a large range of slope angles $\left(\vartheta=21^{\circ}-33^{\circ}\right.$ each degree), it has been possible to estimate theoretically the time $t *$ for all the inclinations. Fig. $5 d$ shows the estimated $t *$ 
(a)

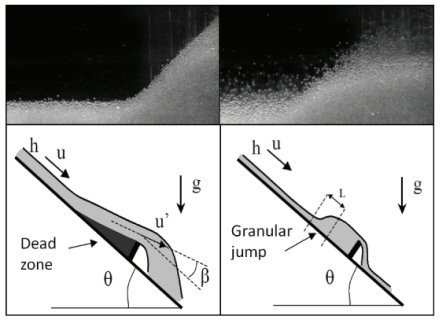

(c)

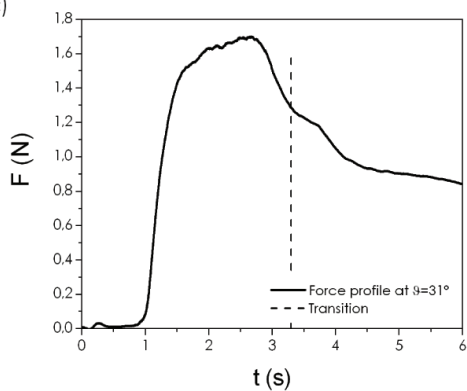

(b)

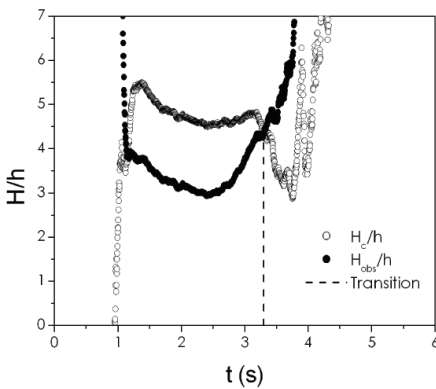

(d)

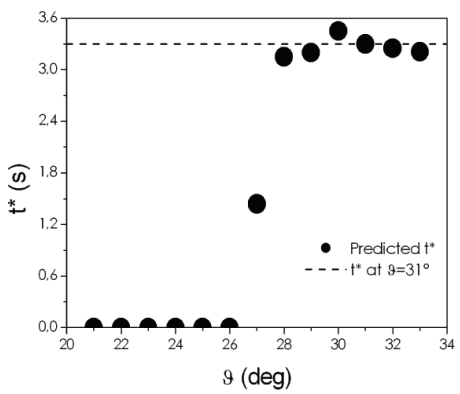

Figure 5: (a) Sketches representing the dead zone and granular jump regimes with respective images corresponding to the two phases occurring during the flow. (b) Comparison between the theoretical ratio $H_{c} / h$ and the experimental ratio $H_{o b s} / h$. The intersection between the two curves indicates the time at which transition should happen. (c) Mean force value $F$ versus the time $t$ at $\vartheta=31^{\circ}$. The dashed line corresponds to the time $t *$ theoretically obtained. (d) Transition time versus the slope angle. Values represent a prediction based on reference measurements data.

versus the slope angle $\vartheta$. According to the prediction results, the transition from dead zone to granular jump regime would not occur for $\vartheta<\vartheta_{\max }$. Beyond the $\vartheta_{\max }$ angle, transitions generates at a time $t$ rather constant and equal to $3.2-3.3 \mathrm{~s}$.

\section{Conclusions}

This work aimed to investigate the behavior of the stagnant zone of a granular avalanche. The present study highlights that this stagnant zone evolves during the flow. First, a triangular quasi-static dead zone generates and coexists with an inertial zone above it. The dead zone reaches a stationary regime before developing into a granular jump (discontinuity in velocity and flow depth). This transition influences the total force acting on the obstacle, so, a better knowledge on the phenomenon contributes to the improvement of the design of passive protection structures. Moreover, analytical models have been developed $[4,5]$ to simulate the 
dynamical behavior of an avalanche interacting with an obstacle. These models are based on the assumption that the parameters of the stagnant zone evolve as mentioned in Section 3.1, without taking into account the dead zone to granular jump transition. So these results additionally allow the improvement of analytical models providing a contribution to the general knowledge on the physics of granular avalanches around an object.

\section{Acknowledgements}

This study received financial support from the European project Dynaval (INTERREG-ALCOTRA). The authors would like to thanks Frederic Ousset for his crucial contribution to the experimental device design and Benoit Chanut for previous inspiring works.

\section{References}

[1] P. Bartelt and B. W. McArdell. Instruments and methods: Granulometric investigation of snow avalanches. Journal of Glaciology, 55:829-833, 2009.

[2] P. Caccamo, B. Chanut, T. Faug, H. Bellot, and F. Naaim-Bouvet. Small-scale experiments to investigate dry granular avalanche dynamics and forces on a wall-like obstacle. Granular Matter, in prep.

[3] B. Chanut. Force exercée par une avalanche granulaire dense sur un obstacle. Application aux digues de protection paravalanche. $\mathrm{PhD}$ thesis, University of Grenoble, 2010.

[4] B. Chanut, T. Faug, and M. Naaim. Time-varying force from dense granular avalanches on a wall. Physical review E, 82(041302):1-12, 2010.

[5] T. Faug, R. Beguin, and B. Chanut. Mean steady granular force on a wall overflowed by free-surface gravity-driven dense flows. Physical Review E, 80(021305):1-13, 2009.

[6] Y. Forterre and O. Pouliquen. Flows of dense granular media. The Annual Review of Fluid Mechanics, 40:1-24, 2008.

[7] GDRMiDi. On dense granular flow. The European Physical Journal E, 14:341-365, 2004.

[8] K. M. Hákonardóttir. The interaction between snow avalanches and dams. PhD thesis, University of Bristol, 2004.

[9] O. Pouliquen. Scaling laws in granular flows down rough inclined planes. Physics of Fluids, 11(3):542-548, 1999.

[10] P.G. Rognon, F. Chevoir, H. Bellot, F. Ousset, M. Naaim, and P. Coussot. Rheology of dense snow flows: inferences from steady state chute-flow experiments. Journal of Rheology, 52(3):729-748, 2008.

[11] B. Salm. Flow, flow transition and run-out distances of flowing avalanches. Annals of Glaciology, 18:221-226, 1993.

[12] B. Sovilla, M. Kern, and M. Schaer. Slow drag in wet-snow avalanche flow. Journal of Glaciology, 56(198):587-592, 2010. 